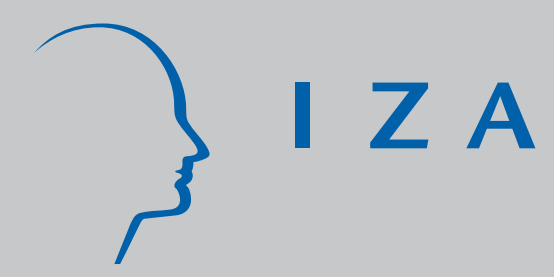

IZA DP No. 2763

Symbolic Values, Occupational Choice, and Economic Development

Giacomo Corneo

Olivier J eanne

April 2007 


\title{
Symbolic Values, Occupational Choice, and Economic Development
}

\author{
Giacomo Corneo \\ Free University of Berlin, \\ CEPR, CESifo and IZA \\ Olivier Jeanne \\ International Monetary Fund \\ and CEPR
}

Discussion Paper No. 2763

April 2007

IZA

P.O. Box 7240

53072 Bonn

Germany

Phone: +49-228-3894-0

Fax: +49-228-3894-180

E-mail: iza@iza.org

Any opinions expressed here are those of the author(s) and not those of the institute. Research disseminated by IZA may include views on policy, but the institute itself takes no institutional policy positions.

The Institute for the Study of Labor (IZA) in Bonn is a local and virtual international research center and a place of communication between science, politics and business. IZA is an independent nonprofit company supported by Deutsche Post World Net. The center is associated with the University of Bonn and offers a stimulating research environment through its research networks, research support, and visitors and doctoral programs. IZA engages in (i) original and internationally competitive research in all fields of labor economics, (ii) development of policy concepts, and (iii) dissemination of research results and concepts to the interested public.

IZA Discussion Papers often represent preliminary work and are circulated to encourage discussion. Citation of such a paper should account for its provisional character. A revised version may be available directly from the author. 


\section{ABSTRACT \\ Symbolic Values, Occupational Choice, and Economic Development ${ }^{*}$}

Channeling human resources into the right occupations has historically been a key to economic prosperity. Occupational choices are not only driven by the material rewards associated with the various occupations, but also by the esteem that they confer. We propose a model of endogenous growth in which occupations carry a symbolic value that makes them more or less attractive; the evolution of symbolic values is endogenous and determined by purposive transmission of value systems within families. The model sheds light on the interaction between cultural and economic development and identifies circumstances under which value systems matter for long-run growth. It shows the possibility of culturally determined poverty traps and offers a framework for thinking about the transition from traditional to modern values.

JEL Classification: D1, O1

Keywords: $\quad$ symbolic values, occupational choice, economic development, long-run growth

Corresponding author:

Giacomo Corneo

Department of Economics

FU Berlin

Boltzmannstr. 20

14195 Berlin

Germany

E-mail: gcorneo@wiwiss.fu-berlin.de

\footnotetext{
* We are grateful to seminar participants at various universities and research institutes for helpful comments. This paper was partly written while Olivier Jeanne was visiting the Department of Economics of Princeton University, whose hospitality is gratefully acknowledged.
} 


\section{Introduction}

Economic take-offs are typically accompanied by pervasive changes in the values endorsed by people. For example, in western Europe the transition from a feudal to a capitalistic mode of production was accompanied by a transition from traditional to modern values. Whereas the former emphasize land possession, religion, and combat skill, the latter praise work, education, and economic achievement. Also in the decades after World War II, considerable changes in values have been documented in rapidly growing countries such as the US, Japan, and western Europe. ${ }^{1}$

The concomitance of value change and economic development raises a fundamental question of causation, one that has been intensely debated for a long time. A whole spectrum of views exists, ranging from the culturalist one, according to which values are the engine of economic growth, to the materialistic view, which confers that role to technology and interprets value change as a mechanical adjustment. Far from being merely academic, the issue of the interplay of culture and economic performance can have profound policy implications. In some areas of the world, mass poverty goes hand in hand with values and norms that are hostile to entrepreneurship and technical progress. Culture may or may not be a crucial factor behind the failure of development policies in countries caught in a poverty trap. And, if values do cause development, assessing their "malleability" could make an important contribution to the design of successful policies.

In the current paper, we develop a tractable model that enables one to study the interrelationship between economic development and value change, with a focus on the value attached to economic activities or occupations. We introduce the concept of "symbolic value" to account for the observation that some personal characteristics, e.g. one's professional activity, seem to be invested with value by human beings. The value associated with those characteristics determines the self-esteem of individuals as well as the esteem that they receive from other individuals. Values are symbolic in the sense of being immaterial: they affect the well-being of individuals, but without altering their consumption of material goods.

Formally, we shall define a value system as a function that maps from a set of judgeable characteristics into a set of index numbers. Thereby, each "judgeable type" is associated with a scalar that represents its value. Value systems are individual-specific and determine

\footnotetext{
${ }^{1}$ Empirical sociologists Inglehart and Baker (2000) refer to this change as to a shift from a value system that emphasized "survival" to one that emphasizes "self-expression".
} 
how much esteem individuals allocate to themselves and others. In turn, self-esteem and the esteem received from others are arguments of an individual's utility function. ${ }^{2}$

Arguably, value systems are heavily influenced by intergenerational transmission within families. ${ }^{3}$ The current paper analyzes the polar case where parents choose the value system of their children so as to maximize their children's expected utility. The choice set for parents is defined so as to capture the fact that it is easier to teach the values one endorses rather than values one has not internalized.

This model of values and value formation is combined with an endogenous growth model in order to highlight the dynamic interaction between culture and economic development. In particular, we address the following questions: Can values affect growth in the long run? How does economic development affect values?

Our growth model has symbolic value attached to occupations. ${ }^{4}$ Following a line of research pioneered by Aghion and Howitt (1992), Baumol (1990), Murphy et al. (1991), and Romer (1990), we posit that occupations diverge in terms of their spillovers on the returns of other occupations. For instance, as pointed out by Baumol (1990) and Murphy et al. (1991), engineers and lawyers may contribute in quite different amounts to technological progress and growth.

The model in the current paper can generate equilibria consistent with either the culturalist view, in which values have a long-run impact on prosperity, or the materialistic one, in which values do not matter. Our main finding relates to the circumstances under which each type of pattern arises as an equilibrium outcome. The culturalist view of development is more likely to be right if the intertemporal elasticity of substitution of consumption is low, predictability of the economic environment is high, and concerns for social ostracism are large.

The intuition is as follows. The intertemporal elasticity of substitution of consumption plays a key role in our model because it determines whether the marginal utility of labor income in terms of consumption increases or decreases with economic development. One the one hand, economic development raises productivity and so increases the

\footnotetext{
${ }^{2}$ Many researchers have studied the economic implications of a concern for social esteem, see Fershtman and Weiss (1998a) for a review of the literature. Caring about the opinion of others may be wired into human beings as the outcome of evolutionary selection, as argued e.g. by Fershtman and Weiss (1998b).

${ }^{3}$ See e.g. Fernandez (2007) for an insightful discussion of the empirical evidence on the impact of parents' culture on female labor force participation.

${ }^{4}$ In medieval towns, one's association with a given craft, often organized in a guild, was a distinct source of pride. Even nowadays, people feel the aura that surrounds various activities, e.g. being a soldier, a scientist or an artist. Moreover, within a given society, its members often attach to the same occupation quite different symbolic values. For example, the entrepreneur is the hero of economic prosperity to some, and the greedy speculator to others. Different societies have throughout history exhibited different attitudes toward various occupations. By way of an example, the clergy in European societies enjoyed much more respect one century ago than today.
} 
wage. On the other hand, it also increases the level of consumption and so decreases the marginal utility in terms of consumption of a given increase in labor income. If the intermporal elasticity of substitution of consumption is larger than one, income differences across occupations eventually weigh so much in terms of utility, that material payoffs eventually dominate career choices. Then, differences in value systems exert no influence on the long-run behavior of the economy. Conversely, if the intertemporal elasticity of substitution of consumption is smaller than unity, symbolic values eventually dominate occupational choice even if pay differences across occupations become arbitrarily large. Uncertainty about the income opportunities of the various occupations enters the picture as soon as individuals are risk averse with respect to self-esteem. Then, optimal socialization decisions may entail value diversification, i.e., transmitting an agnostic attitude towards the symbolic value of occupations. If uncertainty is large enough, individuals progressively abandon strong views about the worth of the various activities and esteem concerns asymptotically evaporate. Fear of social ostracism fosters conformism and can generate multiple value-led equilibria. Concerns for approval by others may dramatically exacerbate the effect of values on long-run growth whenever the intertemporal elasticity of substitution of consumption is smaller than one.

If the intertemporal elasticity of substitution of consumption is low, predictability of the economic environment is high, and concerns for social ostracism are large, culturally determined poverty traps can arise. While economic growth could be fast under a different value system, "wrong" values may have such an adverse effect on the allocation of human resources, that the economy fails to develop in spite of its favorable preconditions in term of physical capital, knowledge, and protection of property rights.

Is there evidence of economies that might have experienced a cultural trap? According to Baumol (1990), two historical examples that exhibit features of a cultural trap are Rome and China. Ancient Rome failed to put into widespread practical use some of the sophisticated technological developments that have been in its possession because of contemptuous attitudes of the upper class towards commerce and industry. By contrast, innovations spread like wildfire in Italy during the Renaissance, a time at which the upper classes had the "right values", i.e., considered commerce and industry honorable activities. Imperial China during the Middle Ages is another example of a society where rent-seeking activities with high prestige delivered larger rewards relative to productive activities and that missed the opportunity of an economic take-off in spite of its high level of technological knowledge. 


\section{Links to the literature}

The current paper is related to two strands of literature, one that studies the impact of norms on economic growth and one that analyzes the formation and transmission of cultural traits.

In the literature on economic growth, Cole et al. (1992) proposed a model where multiple social norms to achieve status can be sustained as an equilibrium. Specifically, they characterized a wealth-is-status equilibrium and an aristocratic equilibrium where birth determines one's position in the social ladder. Growth is faster under the wealth norm because the quest for status creates an additional incentive to accumulate. In contrast to the current paper, status in their paper is sought for instrumental reasons, i.e., to improve one's access to mates. Furthermore, their paper does not deal with the issue of how a norm is selected over others, whereas the current paper determines the values system of society as the outcome of a decentralized process of socialization within families.

The role of prestige attached to occupations plays a key role in Fershtman et al. (1996), who developed a model in which individuals can either accumulate human capital and become managers, or they do not accumulate human capital and become laborers. Accumulating human capital produces knowledge that raises overall productivity, so that economic growth is endogenous. Individuals are posited to care about their occupational status and the status of each occupation is assumed to increase with the average human capital of its members relative to the human capital in the other occupation.

Whereas Fershtman et al. just assume that higher social status is bestowed on the occupation that enhances growth, in our model the esteem of occupations is endogenous and depends on the values that parents transmit to their children. Hence, in our model the symbolic ranking of occupations can but need not mimick their ranking in terms of contribution to overall productivity growth. This is more general and in line with the observation that in many societies higher status is associated with activities that are unlikely to promote economic growth, like the clergy and the military. In our model, the quest for esteem may or may not foster economic growth.

Empirical evidence that culture has a causal effect on economic development has recently been offered by Tabellini (2006). He measures culture by indicators of individual values and beliefs and proposes a method to isolate the exogenous component of culture. Using data on European regions, Tabellini finds that culture is strongly correlated with current economic development, after controlling for education and political institutions. ${ }^{5}$

\footnotetext{
${ }^{5}$ Guiso et al. (2006) offers an excellent discussion of culture as a determinant of economic phenomena and of the empirical methods that can be employed to identify the economic role of culture.
} 
Our model of value transmission is closely related to models of cultural evolution proposed by Bisin and Verdier $(2000,2001),{ }^{6}$ who have studied settings in which parents purposely socialize their children to selected cultural traits. This vertical socialization, along with intragenerational imitation, determines the long-term distribution of cultural traits in the population. Under some conditions, Bisin and Verdier's theory predicts convergence to a culturally heterogeneous population.

Our approach mainly differs from Bisin and Verdier's theory in two respects. First, Bisin and Verdier assume that parents want their children to have the same cultural trait as themselves. They motivate this assumption by the possibility of "imperfect empathy" on the side of parents. This means that parents evaluate their children's actions using their (the parents') preferences. In our theory, by contrast, parents choose the value system of their children so as to maximize the children' utility, and the objects that are transmitted from parents to children are modeled in a different way. Whereas in Bisin and Verdier's theory parents transmit a preference trait, in ours they transmit a value system. The essential property of a value system is that, taking it in conjonction with a course of action, it determines the esteem enjoyed by the individual. In our theory, individuals have preferences over esteem and the usual list of consumption goods. The advantage of modeling socialization to a value system rather than to a preference trait is that one keeps preferences fixed, so that normative analysis based on the Pareto criterion is possible. ${ }^{7}$ The cost of this modeling approach is that one has to add esteem to the standard arguments of the utility function. But also Bisin and Verdier's theory works with an additional argument in the utility function, namely the offspring's preference parameter.

A related appproach has been proposed by Akerlof and Kranton (2000, 2005), whose notion of identity shares some important features with our notion of self-esteem. In their theory, a person's identity is associated with different social categories and how people in these categories should behave. Violating behavioral prescriptions causes a utility loss and may produce responses by others who want to defend their sense of self. We follow Akerlof and Kranton's theory in that we also generalize the utility function so as to include arguments that capture important nonpecuniary motivations of human action. However, we employ a different method to determine the prevailing norms of behavior. Akerlof and Kranton use sociological evidence to formulate assumptions about behavioral prescriptions that are likely to capture important aspects of reality. We derive those prescriptions as

\footnotetext{
${ }^{6}$ Bisin and Verdier's approach to value transmission is applied to trust and economic development by Francois and Zabojnik (2005).

${ }^{7}$ We refer to Corneo and Jeanne (2006) for a study of allocative efficiency in the presence of endogenous values.
} 
part of an equilibrium in a model based on individual optimization under constraints.

A recent paper by Bénabou and Tirole (2006) analyzes issues of identity in a model where people value and invest in beliefs. Differently from our framework, they focus on the individual management of beliefs and the cognitive mechanisms through which it occurs when the individual is unsure of his own deep preferences. Thus, while theirs as well as our paper endogenize identity-related payoffs, the two papers concentrate on different mechanisms of value formation.

\section{Symbolic values}

Our approach to symbolic values is based on four hypotheses. We make no claim of originality, as they have been put forward by plenty of scholars before us.

\section{Postulate 1: Evaluative Attitude}

Individuals pass judgments of approval, admiration, etc., and their opposite upon certain traits, acts, and outcomes.

Individuals evaluate bundles of judgeable characteristics, also referred to as types. An individual's value system is a description of that evaluation. Formally, we shall define the value system of an individual as a function that maps the set of judgeable individual characteristics onto the real line. We take the set of judgeable individual characteristics as exogenously given. ${ }^{8}$

We think of the evaluation of types as an essentially relative procedure by which granting more value to a type implies that less value is attributed to the remaining ones. A special case is one where the individual ranks all types and the symbolic value that the individual associates with any particular type is that type's rank. Since the total number of ranks is given, assigning a higher value to a given type would then imply that a lower value is associated to other types. However, we do not want to restrict value systems to be rank-dependent because people' s judgements seem to entail more than rank information: two types that rank one after the other may be close or far apart in terms of their symbolic values and that difference should be captured by two different value systems. Therefore, in order to capture both the relative dimension of values and value differences that do not stem from differences in rank, we normalize the total amount of value that an individual associates with all types to a constant; the allocation of that amount to the various types is then defined as the individual's value system.

\footnotetext{
${ }^{8} \mathrm{~A}$ similar approach is adopted in the models of cultural evolution and identity. There, the existence of a culturally relevant trait and that of a social category are taken as given.
} 


\section{Postulate 2: Social approbativeness}

Individuals desire a good opinion of themselves on the part of other people.

The relevant human environment for approbativeness may be an individual's family, friends, colleagues, neighbors, or society at large. The desired approbation may involve a fear of contempt or indifference, or a craving for the interest, approval, praise, or admiration of others. The current paper merely examines the esteem received by individuals from society at large.

\section{Postulate 3: Self-approbativeness}

Individuals have a desire for self-esteem.

The desire for a pleasing idea of oneself presupposes self-consciousness. Humans are both actors and spectators of what they are doing. Since they are evaluative beings, they also judge themselves. Actually, at least some modest measure of self-esteem seems to be indispensable to an endurable existence.

\section{Postulate 4: Consistency}

The standards of approbation or disapprobation which the individual applies to himself are the same as those which he applies to other people.

This postulate corresponds to the rule of judging yourself as you would judge of others. While psychologists have identified ways of self-deception, i.e., methods that individuals adopt to manipulate their self-image, in the main individuals are subject to a consistency constraint. It is difficult to systematically approve in oneself acts which one condemns in others, and when one does so, his fellows are quick to point out the inconsistency.

People's well-being is supposed to depend upon both self-esteem and the esteem received by other people, along with consumption of goods and services. When choosing a course of action, individuals compare the pecuniary return of actions and the esteem they carry.

Value systems can form within various socialization structures. This paper concentrates on the benchmark case of socialization by altruistic parents. A more general approach would have parents compete with other agencies of socialization like the school, the church, and the children's peers. We discuss this topic in the concluding Section.

\section{The deterministic model}

Time is discrete $t=0,1, . . \infty$. There are overlapping generations of individuals living for two periods. In their first period, individuals are socialized by their parents; in their 
second period, they produce, consume, and socialize their children. Specifically, at each time $t$ there is a continuum of mass 1 of adults, indexed by $i_{t} \in[0,1]$, and a continuum of children, $i_{t+1} \in[0,1]$. Individual $i_{t}$ is the parent of individual $i_{t+1}$. Adult individuals consume one homogeneous nonstorable good, which is used as the numeraire. Individuals have common preferences and specialize in one of two activities or occupations, referred to as $a$ and $b$. Each parent chooses his occupation so as to maximize his utility, and the values of his child so as to maximize his child's expected utility.

A value system associates a non-negative index $v(x)$ to occupation $x \in\{a, b\}$. We impose the normalization

$$
v(a)+v(b)=1,
$$

so that the value of an activity relative to the alternative, $v(x)-v\left(x^{\prime}\right)$, is between -1 and +1 . Equation (1) can also be interpreted as setting an upper bound to the intensity of value concerns.

Each individual is equipped with a value system. We denote the value system of an individual $i$ that was socialized in $t-1$ and is active in $t$ by $\left\{v\left(a, i_{t}\right), v\left(b, i_{t}\right)\right\}$.

For a parent it may be difficult to transmit values that are very different from his own. We therefore assume that a parent $i_{t}$ chooses the values of his child subject to the constraint

$$
v\left(a, i_{t+1}\right) \in\left[v\left(a, i_{t}\right)-\eta, v\left(a, i_{t}\right)+\eta\right] \cap[0,1],
$$

where parameter $\eta \in(0,1]$ captures the maximum distance between the parent's values and those of the child. This assumption can also be interpreted as one about the costs to a parent of socializing his child to values that the parent does not endorse.

Values determine the individuals' self-esteem and social esteem. In the current model we concentrate on the role of self-esteem; social esteem will be introduced in the final part of the paper.

An individual's self-esteem is the value of his occupation according to his value system: $v\left(x\left(i_{t}\right), i_{t}\right)$, where $x\left(i_{t}\right) \in\{a, b\}$ denotes the individual's occupation.

Individuals care about consumption and esteem. The utility of individual $i_{t}$ is separable in consumption and esteem concerns and given by,

$$
U\left(i_{t}\right)=f\left(y_{t}\left(x\left(i_{t}\right)\right)\right)+h\left(v\left(x\left(i_{t}\right), i_{t}\right),\right.
$$

where $f\left(y_{t}\left(x\left(i_{t}\right)\right)\right)=\frac{\left(y_{t}\left(x\left(i_{t}\right)\right)\right)^{1-\sigma}}{1-\sigma}, \sigma>0$, captures utility from consumption and $h\left(v\left(x\left(i_{t}\right), i_{t}\right)\right)=$ $\lambda v\left(x\left(i_{t}\right), i_{t}\right)$ captures utility from esteem; $\lambda>0$ parametrizes the strength of the selfesteem concern. Assuming utility to be linear in esteem is only for the sake of simplicity; this assumption will be relaxed in Section 7 . 
Consumption equals the individual's income. The incomes of occupations are denoted by $y_{t}(x), x \in\{a, b\}$, and determined in the labor market according to

$$
y_{t}(x)=A_{t} Y_{x}\left(n_{t}\right)
$$

where $n_{t}$ is the number of individuals practicing occupation $a$ at time $t$. We assume $Y_{a}^{\prime}<0$ and $Y_{b}^{\prime}>0$, and that $Y_{a}(\cdot)$ and $Y_{b}(\cdot)$ are bounded and equal for a value of $n$ denoted by $n^{*} \in(0,1)$. These assumptions can be viewed as a reduced-form model of a competitive labor market under decreasing returns to each occupation.

Economy-wide productivity $A$ evolves according to

$$
A_{t+1}=\left(1+g\left(n_{t}\right)\right) A_{t}
$$

with $g(0)>0$ and $g^{\prime}>0$. Thus, $a$ is the growth-inducing occupation.

Equations (3) and (4) can be seen as a reduced-form model of endogenous growth. In the Appendix, we show how it can be derived from a full-fledged model based on the distinction between traditional sectors and modern sectors that generate knowledge spillovers.

An equilibrium is defined as

-a distribution of values and occupations at each time, $\left(v\left(a, i_{t}\right)\right)_{i_{t} \in[0,1]}$ and $\left(x\left(i_{t}\right)\right)_{i_{t} \in[0,1]}$, -a productivity path $\left(A_{t}\right)$,

-a path for the material payoffs $\left(y_{t}(a)\right.$ and $\left.y_{t}(b)\right)$,

such that:

-for all $i_{t}$, the occupation $x\left(i_{t}\right)$ maximizes $U\left(i_{t}\right)$ conditional on $y_{t}(x)$ and $v\left(x, i_{t}\right)$, $x=a, b$,

-for all $i_{t}$, the values $v\left(a, i_{t+1}\right), v\left(b, i_{t+1}\right)$ maximize $U\left(i_{t+1}\right)$ subject to $(2)$,

-equations (3) and (4) apply.

The initial conditions are given by a distribution of values for the initial generation, $\left(v\left(a, i_{0}\right)\right)_{i_{0} \in[0,1]}$ and an initial productivity level $A_{0}$. Without restriction of generality, we assume that $v\left(a, i_{0}\right)$ is nonincreasing with $i_{0}$.

\section{Values and occupations in the short run}

In a short-run equilibrium, the values and occupational choices of one generation, $v\left(a, i_{t+1}\right)$ and $n_{t+1}$, are endogenously determined, taking as given the previous generation's values and occupational choices.

Let $\bar{v}\left(a, i_{t+1}\right) \equiv \operatorname{Inf}\left\{v\left(a, i_{t}\right)+\eta, 1\right\}$ and $\underline{v}\left(a, i_{t+1}\right) \equiv \operatorname{Sup}\left\{v\left(a, i_{t}\right)-\eta, 0\right\}$, so that parent $i_{t}$ chooses the values of his child in the interval $\left[\underline{v}\left(a, i_{t+1}\right), \bar{v}\left(a, i_{t+1}\right)\right]$. In a perfect-foresight 
equilibrium, each parent knows the occupation of his offspring. Hence, the parent puts the maximal symbolic value on that occupation, since this increases his offspring's selfesteem without affecting other determinants of his utility. Formally, an equilibrium path necessarily satisfies

$$
\begin{aligned}
& v\left(a, i_{t+1}\right)=\bar{v}\left(a, i_{t+1}\right) \quad \text { if } \quad x\left(i_{t+1}\right)=a, \\
& v\left(a, i_{t+1}\right)=\underline{v}\left(a, i_{t+1}\right) \quad \text { if } \quad x\left(i_{t+1}\right)=b .
\end{aligned}
$$

If $\eta$ is sufficiently large, parents are not constrained by their own values in choosing their children's values, i.e. $\bar{v}\left(a, i_{t}\right)=1$ and $\underline{v}\left(a, i_{t}\right)=0$. Then, in an interior equilibrium where both occupations are chosen by a strictly positive mass of individuals, the two occupations must yield the same income. Otherwise a parent would be able to increase his child's welfare by putting all the symbolic value in the activity yielding the highest income. Given our assumptions on $Y_{a}(\cdot)$ and $Y_{b}(\cdot)$, there is a unique equililbrium $n_{t+1}=n^{*}$ and a corner equilibrium cannot exist.

Of course, the outcome $n_{t+1}=n^{*}$ is identical to the one obtained in a model where $\lambda=0$, i.e. agents do not care about esteem. Therefore, if $\eta$ is large, values do not affect the allocation of manpower to occupations and exert no influence on the growth rate of the economy.

In the rest of the paper we mainly concentrate on the case where $\eta$ is small, so that parents may be constrained by their own values when choosing their children's values. ${ }^{9}$ In this case, the material payoffs $A_{t+1} Y_{a}\left(n_{t+1}\right)$ and $A_{t+1} Y_{b}\left(n_{t+1}\right)$ could be different in equilibrium because some parents are unable to teach their children a sufficiently high value for the activity with the highest material payoff.

As noticed above, $\bar{v}\left(a, i_{t+1}\right)$ and $\underline{v}\left(a, i_{t+1}\right)$ are the only possible equilibrium values of individual $i_{t+1}$ for occupation $a$. His parent will opt for $v\left(a, i_{t+1}\right)=\bar{v}\left(a, i_{t+1}\right)$ if

$$
f\left(A_{t+1} Y_{a}\left(n_{t+1}\right)\right)+\lambda \bar{v}\left(a, i_{t+1}\right) \geq f\left(A_{t+1} Y_{b}\left(n_{t+1}\right)\right)+\lambda\left(1-\underline{v}\left(a, i_{t+1}\right)\right) .
$$

The left-hand-side of this inequality is implicitly increasing with $v\left(a, i_{t}\right)$ whereas the righthand-side is decreasing with $v\left(a, i_{t}\right)$. Hence, there exists a critical level for $v\left(a, i_{t}\right)$ such that only parents with a higher value for occupation $a$ choose $\bar{v}\left(a, i_{t+1}\right)$ rather than $\underline{v}\left(a, i_{t+1}\right)$. We denote this critical level by $\widehat{v}\left(n_{t+1}\right)$. Notice that the critical threshold $\widehat{v}\left(n_{t+1}\right)$ is strictly increasing with $n_{t+1}$. Intuitively, if the size of occupation $a$ is predicted to be larger, its

\footnotetext{
${ }^{9}$ Notice that the interval $\left[\underline{v}\left(a, i_{t+1}\right), \bar{v}\left(a, i_{t+1}\right)\right]$ depends on $v\left(a, i_{t}\right)$.
} 
income will be lower and choosing occupation $a$ becomes optimal only for individuals whose parents have a stronger symbolic preference for occupation $a$.

The equilibrium level of $n_{t+1}$ is then solution to the fixed-point problem that the number of parents with $v\left(a, i_{t}\right) \geq \widehat{v}\left(n_{t+1}\right)$ should be equal to $n_{t+1}$. There is at least one solution by standard fixed-point theorems. There cannot be more than one solution since $\widehat{v}\left(n_{t+1}\right)$ is increasing with $n_{t+1}$.

Therefore, a short-run equilibrium exists and is unique. Values and occupations satisfy (5) and (6); all individuals for whom (7) applies, choose occupation $a$ and the remaining individuals choose occupation $b$.

To illustrate, let individuals be ordered according to their parents' values:

$$
i_{t}<j_{t} \Rightarrow v\left(a, i_{t}\right) \geq v\left(a, j_{t}\right) .
$$

Thus, individuals with lower index have parents who put more value on occupation $a$. If $v\left(a, i_{t}\right)$ is continuous in $i_{t}$, the net benefit from choosing occupation $a$ can be expressed as

$$
B_{t+1}\left(n_{t+1}\right)=f\left(A_{t+1} Y_{a}\left(n_{t+1}\right)\right)+\lambda \bar{v}\left(a, n_{t+1}\right)-f\left(A_{t+1} Y_{b}\left(n_{t+1}\right)\right)-\lambda\left(1-\underline{v}\left(a, n_{t+1}\right)\right)
$$

and $B_{t+1}^{\prime}<0$, so that the equilibrium is unique. If $B_{t+1}(0)>0$ and $B_{t+1}(1)<0$, the equilibrium must be interior and both occupations are chosen by a strictly positive mass of individuals; the equilibrium size of occupation $a$ is the unique root of $B_{t+1}\left(n_{t+1}\right)=0$. However, the equilibrium can also be a corner solution in which all individuals choose occupation $a\left(n_{t+1}=1\right.$ and $\left.B_{t+1} \geq 0\right)$ or $b\left(n_{t+1}=0\right.$ and $\left.B_{t+1} \leq 0\right)$. Notice that corner solutions could not arise in the case of a sufficiently large $\eta$.

\section{Growth and values in the long run}

How do values evolve in the long run? The following result describes the evolution of the distribution of values within the population.

Proposition 1 Values are dynastic: if individual $i_{t}$ puts more value than individual $j_{t}$ on occupation a, then this will be true of all their descendants:

$$
v\left(a, i_{t}\right) \geq v\left(a, j_{t}\right) \quad \Longrightarrow \quad \forall t^{\prime}>t, \quad v\left(a, i_{t^{\prime}}\right) \geq v\left(a, j_{t^{\prime}}\right) .
$$

Proof. We prove that if $i_{t}$ puts more value on $a$ than $j_{t}$ then this is also true of their children $i_{t+1}$ and $j_{t+1}$. Then this will be true, by forward induction, of all their descendants. It is clear from the socialization condition (7) that if $v\left(a, i_{t}\right) \geq v\left(a, j_{t}\right)$, it 
cannot be optimal for $i_{t}$ to transmit values that induce his child to practice $b$ while $j_{t}$ does the opposite. So either $v\left(a, i_{t+1}\right)=\bar{v}\left(a, i_{t+1}\right)$ and $v\left(a, j_{t+1}\right)=\bar{v}\left(a, j_{t+1}\right)$, or $v\left(a, i_{t+1}\right)=$ $\bar{v}\left(a, i_{t+1}\right)$ and $v\left(a, j_{t+1}\right)=\underline{v}\left(a, j_{t+1}\right)$, or $v\left(a, i_{t+1}\right)=\underline{v}\left(a, i_{t+1}\right)$ and $v\left(a, j_{t+1}\right)=\underline{v}\left(a, j_{t+1}\right)$. In all three cases one has $v\left(a, i_{t+1}\right) \geq v\left(a, j_{t+1}\right)$. QED

This result, combined with the assumption that $v\left(a, i_{0}\right)$ is nonincreasing with $i_{0}$, implies that $v\left(a, i_{t}\right)$ is nonincreasing with $i_{t}$ at all times $t$.

We now turn to the central issue of this paper, namely the interplay of values and economic outcomes in the long term. Again, we take as a benchmark the case where individuals are not concerned about values, i.e., $\lambda=0$, and ask whether values can make a difference with respect to long-run growth.

If $\lambda=0$, then the arbitrage condition $y_{t}(a)=y_{t}(b)$ applies for all $t$, implying $Y_{a}\left(n_{t}\right)=$ $Y_{b}\left(n_{t}\right)$ and $n_{t}=n^{*}$. Then, as shown by (4), the growth rate is constant and equal to $g\left(n^{*}\right)$.

In order to assess the role of values in the case $\lambda>0$, the concept of "family specialization" is useful. We shall say that a family $i$ is specialized in activity $x$ at time $t$ if for all $t^{\prime} \geq t$, individual $i_{t^{\prime}}$ practices this occupation and has all symbolic value invested in this occupation, i.e.,

$$
\forall t^{\prime} \geq t, x\left(i_{t^{\prime}}\right)=x \text { and } v\left(x\left(i_{t^{\prime}}\right), i_{t^{\prime}}\right)=1
$$

We denote by $s_{t}(x)$ the number of families specialized in occupation $x$ at time $t$.

We are now ready to establish the following fact:

Proposition 2 Assume $\sigma<1$. Then, the families asymptotically specialize themselves in the same way as in the value-less equilibrium:

$$
\begin{aligned}
& \lim _{t \rightarrow+\infty} s_{t}(a)=n^{*}, \\
& \lim _{t \rightarrow+\infty} s_{t}(b)=1-n^{*} .
\end{aligned}
$$

The long-run growth rate is the same as in the value-less equilibrium, $g\left(n^{*}\right)$.

Proof. On the equilibrium path one necessarily has,

$$
A_{t}^{1-\sigma} \frac{\left|Y_{a}\left(n_{t}\right)^{1-\sigma}-Y_{b}\left(n_{t}\right)^{1-\sigma}\right|}{1-\sigma} \leq \lambda
$$

otherwise all individuals, irrespective of their values, would choose the same occupation, namely the one with the higher pecuniary payoff. Since $n^{*} \in(0,1)$, the pecuniary payoff 
of the chosen occupation would then be lower than the one of the other occupation, a contradiction. Hence, the condition (8) must hold.

Condition (8),$\sigma<1$ and $\lim _{t \rightarrow+\infty} A_{t}=+\infty$ imply that $\lim _{t \rightarrow+\infty} \mid Y_{a}\left(n_{t}\right)^{1-\sigma}-$ $Y_{b}\left(n_{t}\right)^{1-\sigma} \mid=0$ on the equilibrium path. This means that

$$
\lim _{t \rightarrow+\infty} n_{t}=n^{*}
$$

This implies that the long-run growth rate is the same as in the value-less equilibrium. This property also implies that for any $\varepsilon>0$, there is a $T$ such that for all $t \geq T$, $n^{*}-\varepsilon<n_{t}<n^{*}+\varepsilon$. The dynasties $i_{t} \leq n^{*}-\varepsilon$ practice activity $a$ after time $T$ and invest symbolic value in this activity as quickly as they can, endowing activity $a$ with all the symbolic value after time $T+1 / \eta$ at the latest. The dynasties $j_{t} \geq n^{*}+\varepsilon$ practice activity $b$ after time $T$ and have endowed this activity with all the symbolic value after time $T+1 / \eta$ at the latest. This proves the first part of the Proposition. QED

Proposition 2 identifies a simple condition under which the materialistic view is asymptotically correct. If the intertemporal elasticity of substitution of consumption is larger than unity, the value system of a society at a given point in time will not have any effect on the rate of economic growth that that society will experience in the long run. By the same token, two economies with the same fundamentals but with very different value systems will converge to the same growth rate. Thus, if convergence to the asymptotic growth rate is fast, there may be no point for policy makers to try to influence people's values.

As a counterpart of the materialistic result, we establish:

Proposition 3 Assume $\sigma>1$. Then, the families asymptotically specialize themselves according to

$$
\begin{aligned}
\lim _{t \rightarrow+\infty} s_{t}(a) & =\bar{n}, \\
\lim _{t \rightarrow+\infty} s_{t}(b) & =1-\bar{n},
\end{aligned}
$$

where $\bar{n}$ could be lower or higher than $n^{*}$. The asymptotic growth rate $g(\bar{n})$ could be higher or lower than $g\left(n^{*}\right)$, depending on the initial distribution of values. Skewing the initial distribution of values toward occupation a increases the long-run growth rate.

Proof. Let us introduce a variable $\widetilde{v}_{t}$, implicitly defined by

$$
\frac{A_{t+1}^{1-\sigma}}{\sigma-1} \max _{n}\left|Y_{a}(n)^{1-\sigma}-Y_{b}(n)^{1-\sigma}\right|=\lambda\left(2 \widetilde{v}_{t}-1\right),
$$


where $\sigma>1$. Since the l.h.s of (10) is strictly positive and converges to zero as $A_{t+1}$ goes to infinity, $\widetilde{v}_{t}$ converges to $1 / 2$ from above.

Suppose for a moment $v\left(a, i_{t}\right)=\widetilde{v}_{t}$. Then, the r.h.s. of (10) is the utility gain for $i_{t}$ from choosing occupation $a$ rather than $b$ which is due to their different symbolic values. If $\eta$ were 0 , this would also be the corresponding gain for $i_{t+1}$. The l.h.s. of (10) is the maximum utility gain that $i_{t+1}$ could possibly obtain from choosing one occupation over the other because of their different income.

Now, let $v\left(a, i_{t}\right)$ be arbitrary. If $v\left(a, i_{t}\right) \geq \widetilde{v}_{t}$, parent $i_{t}$ finds it optimal to transmit $\bar{v}\left(a, i_{t+1}\right)$ to his offspring, i.e., $v\left(a, i_{t+1}\right)=\bar{v}\left(a, i_{t+1}\right) \geq v\left(a, i_{t}\right) \geq \widetilde{v}_{t}$. Since $\widetilde{v}_{t}$ monotonically converges from above, $\widetilde{v}_{t+1} \leq \widetilde{v}_{t}$. By transitivity, $v\left(a, i_{t+1}\right) \geq \widetilde{v}_{t+1}$. Hence, also individual $i_{t+1}$ optimally socializes his offspring to occupation $a$ and the same applies to all future generations. Therefore, all families that satisfy $v\left(a, i_{t}\right) \geq \widetilde{v}_{t}$ specialize in activity $a$ in finite time.

A symmetric argument applies to activity $b$. The parents with $v\left(a, i_{t}\right) \leq 1-\widetilde{v}_{t}$ optimally transmit $\underline{v}\left(a, i_{t+1}\right)$ to their children, who in turn satisfy $v\left(a, i_{t+1}\right) \leq 1-\widetilde{v}_{t+1}$ and so on. These families specialize in activity $b$ in finite time.

Since $\widetilde{v}_{t}$ asymptotically converges to $1 / 2$, all families must fall in one of the two categories sooner or later, i.e.

$$
\lim _{t \rightarrow+\infty} s_{t}(a)+s_{t}(b)=1,
$$

which proves the first part of the Proposition.

We then illustrate the multiplicity of long-run growth rates by looking at the range of $\bar{n}$ that can be sustained in steady growth equilibria. Let us assume that at time 0 a fraction $\bar{n}$ of individuals has all symbolic value in occupation $a$ and a fraction $1-\bar{n}$ of individuals has all the value in occupation $b$. Then, this state of affairs persists for ever if a parent specialized in activity $x$ has no incentive to shift the values of his offspring to the other activity, i.e.

$$
\frac{A_{1}^{1-\sigma}}{1-\sigma} Y_{x}(\bar{n})^{1-\sigma}+\lambda \geq \frac{A_{1}^{1-\sigma}}{1-\sigma} Y_{x^{\prime}}(\bar{n})^{1-\sigma}+\lambda \eta
$$

which is true if,

$$
\frac{A_{1}^{1-\sigma}}{\sigma-1}\left|Y_{a}(\bar{n})^{1-\sigma}-Y_{b}(\bar{n})^{1-\sigma}\right| \leq \lambda(1-\eta) .
$$

This condition ensures that the parents maximize their children's welfare by transmitting their own values. For $\eta<1$ it defines a nondegenerate interval for $\bar{n}$ that includes $n^{*}$ in its interior. The asymptotic growth rate is $g(\bar{n})$.

We now come to the last part of the Proposition. Given some initial distribution $v\left(a, i_{0}\right)$, let us skew it toward occupation $a$ in the sense of (weakly) increasing $v\left(a, i_{0}\right)$ for 
all individuals $i_{0}$, keeping $v\left(a, i_{0}\right)$ decreasing with $i_{0}$. Let $v^{\prime}\left(a, i_{0}\right)$ denote the resulting distribution. Let $A_{t}^{\prime}$ denote the productivity path under the distribution $v^{\prime}\left(a, i_{0}\right)$ and let $\widetilde{v}_{t}^{\prime}$ be implicitly defined by

$$
\frac{A_{t+1}^{\prime 1-\sigma}}{\sigma-1} \max _{n}\left|Y_{a}(n)^{1-\sigma}-Y_{b}(n)^{1-\sigma}\right|=\lambda\left(2 \widetilde{v}_{t}^{\prime}-1\right) .
$$

Furthermore, define $\Omega_{t} \equiv\left\{i \mid v\left(a, i_{t}\right) \geq \widetilde{v}_{t}\right\}$ and $\Omega_{t}^{\prime} \equiv\left\{i \mid v^{\prime}\left(a, i_{t}\right) \geq \widetilde{v}_{t}^{\prime}\right\}$.

Consider the occupational choices of generation 0. Since $v^{\prime}\left(a, i_{0}\right) \geq v\left(a, i_{0}\right)$ for all $i \in[0,1]$, the corresponding equilibrium will have $n_{0}^{\prime} \geq n_{0}$. It follows that $A_{1}^{\prime} \geq A_{1}$, so that $\widetilde{v}_{0}^{\prime} \leq \widetilde{v}_{0}$. Therefore, $\Omega_{0} \subseteq \Omega_{0}^{\prime}$. Proceeding forwards, $v^{\prime}\left(a, i_{t}\right) \geq v\left(a, i_{t}\right)$ for all $i \in[0,1]$ will also be true for all $t>0$, so that $\Omega_{t} \subseteq \Omega_{t}^{\prime}$ always holds. This implies that the number of families that eventually specialize in occupation $a$ is larger under the distribution $v^{\prime}\left(a, i_{0}\right)$. Hence, also the long-term growth rate is larger under that distribution. QED

Propositions 2 and 3 can be related to Keynes' famous speculations on the "Economic Possibilities for Our Grandchildren", where he conjectured that if economic growth continues long enough, material needs will be satiated and human beings will devote their energies to non-economic purposes (Keynes, 1972). This is the case, in the current model, if occupational choices are increasingly influenced by values as $A_{t}$ increases to infinity; then, in the limit, all individuals choose the occupation with the highest symbolic value irrespective of material payoffs.

It is noteworthy that our proof of the long-run dominance of symbolic rewards does not rely on a bounded utility from material consumption. If the utility from consumption is logarithmic $(\sigma=1)$, the long-run growth rate remains influenced by values. ${ }^{10}$

A major insight from Proposition 3 concerns the possibility of a culturally determined poverty trap. Consider two economies with the same preferences and the same initial technology. If the intertemporal elasticity of substitution of consumption is smaller than one, a small difference with respect to their initial value systems may cause their income ratio to asymptotically converge to zero or infinity. In this case, values matter for long-run growth, but they do not necessarily foster growth. Their long-term impact depends upon the shape of the value system of society at the initial period.

\footnotetext{
${ }^{10}$ Then, the range of possible steady states $\bar{n}$ satisfies $\left|\log \left(Y_{a}(\bar{n}) / Y_{b}(\bar{n})\right)\right| \leq \lambda(1-\eta)$. Proposition 3 can also be generalized to utility functions $f(\cdot)$ for which there exists some minimal consumption level $c$ such that $\sigma(y) \geq 1$ for all $y>c$, where $\sigma(y) \equiv-f^{\prime \prime}(y) y / f^{\prime}(y)$ is not restricted to be constant. Also Proposition 2 can be generalized in a similar fashion.
} 


\section{Uncertainty, values, and growth}

Proposition 3 identifies circumstances under which culture can be decisive for an economic take-off and thus policies that influence values can have a major impact on development and prosperity. We now scrutinize to what extent this assessment remains valid if one allows income uncertainty to enter the picture. Whereas the basic model of the previous Section posits that parents know with certainty the income levels that their offsprings can achieve in each occupation, technology shocks may introduce a considerable amount of uncertainty about those variables. That uncertainty could result in socialization strategies that systematically differ from those derived in the deterministic model, with distinctive consequences for long-run growth.

In order to study the role of economic uncertainty, we modify equation (3) so as to include an aggregate shock in the determination of future incomes, i.e.

$$
y_{t}(x)=\left(1+\delta_{t}(x) \chi\right) A_{t} Y_{x}\left(n_{t}\right)
$$

where $\delta_{t}(a)=-\delta_{t}(b)=1$ with probability $1 / 2$ and $\delta_{t}(a)=-\delta_{t}(b)=-1$ with probability $1 / 2$, and $\chi \in[0,1)$ is the size of the uncertainty. ${ }^{11}$ We assume that uncertainty about $\delta_{t}(x)$ is resolved between period $t-1$ and period $t$ : whereas individuals have incomplete information when they select the value system of their children, they face no uncertainty when they choose their own occupation.

When choosing his offspring's values, a parent $i_{t-1}$ maximizes the expected utility of his child,

$$
E\left(f\left(y_{t}\left(x\left(i_{t}\right)\right)\right)+h\left(v\left(x\left(i_{t}\right), i_{t}\right)\right) .\right.
$$

As in the deterministic model, we posit $f\left(y_{t}\left(x\left(i_{t}\right)\right)\right)=\frac{\left(y_{t}\left(x\left(i_{t}\right)\right)\right)^{1-\sigma}}{1-\sigma}, \sigma>0$. While we also maintain the assumption that $h\left(v\left(x\left(i_{t}\right), i_{t}\right)\right.$ is bounded and satisfies $h(0)=0, h^{\prime}>0$, we now posit $h^{\prime \prime}<0$, i.e., the utility from self-esteem is concave. Below, we comment on the linear case.

Before studying the model economy with values, let us consider the benchmark case where values do not matter. If $h^{\prime}=0$, the arbitrage condition $y_{t}(a)=y_{t}(b)$ applies for all $t$, implying $\left(1+\delta_{t}(a) \chi\right) Y_{a}\left(n_{t}\right)=\left(1+\delta_{t}(b) \chi\right) Y_{b}\left(n_{t}\right)$. If $\delta_{t}(a)=1$, then this condition is met by a unique and time-invariant $n_{t}$, denoted by $n^{\alpha}(\chi)$, where $\alpha$ denotes the corresponding state of the world, $n^{\alpha}$ is strictly increasing in $\chi$, and $n^{\alpha}(0)=n^{*}$. If $\delta_{t}(b)=1$, the size of sector $a$ is given by $n^{\beta}(\chi)$, where $\beta$ denotes the corresponding state of the world, $n^{\beta}$ is strictly decreasing in $\chi$, and $n^{\beta}(0)=n^{*}$.

\footnotetext{
${ }^{11}$ The model of the previous Section can be viewed as the special case of the current one in which $\chi=0$.
} 
Absent a concern for esteem, in equilibrium $n_{t}$ takes the value $n^{\alpha}(\chi)$ in states $\alpha$ and value $n^{\beta}(\chi)$ in states $\beta$. The growth rate varies accordingly. The expected growth rate is time-invariant and equal to $\frac{1}{2}\left[g\left(n^{\alpha}(\chi)\right)+g\left(n^{\beta}(\chi)\right)\right]$.

Turn now to the economy with values, i.e., $h^{\prime}>0$. By a continuity argument, if $\chi>0$ but sufficiently small, then the equilibrium properties derived in the case $\chi=0$ examined in Sections 4-6 carry over to the stochastic model of this Section. Specifically, provided that $\eta<1$, values can make a difference with respect to long-run growth if and only if $\sigma>1$. We are now going to show that if $\chi$ is sufficiently large, this possibility vanishes, i.e., the materialistic view holds true also if the intertemporal elasticity of substitution of consumption is smaller than one.

Consider the socialization problem at the individual level. When individual $i_{t}$ chooses its occupation conditional on his values, three cases can a priori occur: the individual chooses activity $a$ irrespective of his income opportunities, he chooses activity $b$ irrespective of his income opportunities, or he chooses activity $a$ if and only if $y_{t}(a)>y_{t}(b)$.

In order to prepare for the next result, suppose for the moment being that $\eta=1$, so that parents are free to choose values in the entire $[0,1]$ interval. Then, individual $i_{t-1}$ optimally selecting the values of his child chooses one of the following strategies: values specialization, i.e. the parent endows one occupation with all the value and the child chooses that activity with probability one; or, values diversification, i.e. the parent endows each occupation with the same value and the child chooses the occupation with the highest income.

To ascertain when each strategy is optimal, let us compute the child's expected utility under each strategy. Let $y_{t}^{\omega}(x), \omega \in\{\alpha, \beta\}$, denote the income of activity $x$ in state $\omega$ at time $t$. Then, specialization in activity $a$ is an option if putting all the symbolic value on that occupation induces the child to choose this occupation even if it yields a lower pecuniary payoff, that is if,

$$
\max _{\omega}\left\{f\left(y_{t}^{\omega}(b)\right)-f\left(y_{t}^{\omega}(a)\right)\right\}<h(1) .
$$

If this condition is satisfied, the parent can induce his child to choose occupation $a$ by putting all the value on this occupation. Analogously, if

$$
\max _{\omega}\left\{f\left(y_{t}^{\omega}(a)\right)-f\left(y_{t}^{\omega}(b)\right)\right\}<h(1),
$$

the parent can induce his child to choose occupation $b$ by putting all the value on this occupation.

If condition (13) is satisfied and the parent chooses $v\left(a, i_{t}\right)=1$, his child's expected utility equals

$$
\frac{1}{2}\left[f\left(y_{t}^{\alpha}(a)\right)+f\left(y_{t}^{\beta}(a)\right)\right]+h(1) .
$$


If condition (14) is satisfied and the parent chooses $v\left(b, i_{t}\right)=1$, his child's expected welfare is

$$
\frac{1}{2}\left[f\left(y_{t}^{\alpha}(b)\right)+f\left(y_{t}^{\beta}(b)\right)\right]+h(1) .
$$

Since we have supposed $\eta=1$, the parent can always select values that induce his offspring to choose the occupation with the largest material reward. Then, two cases are possible. Either there is an activity that delivers the higher income independently of the state of the world, or each activity maximizes income in a different state. In the first case, there is no point in diversifying values: the parent will invest all the symbolic value in the activity that dominates the other one in terms of income. In the second case, the child's expected utility amounts to

$$
\frac{1}{2}\left[\max _{x} f\left(y_{t}^{\alpha}(x)\right)+\max _{x} f\left(y_{t}^{\beta}(x)\right)\right]+\frac{1}{2}\left[h\left(v\left(a, i_{t}\right)\right)+h\left(1-v\left(a, i_{t}\right)\right)\right],
$$

which only depends on values if $h$ is nonlinear. Whereas in the linear case the solution to the socialization problem is not unique, under our assumption $h^{\prime \prime}<0$, the child's expected utility is uniquely maximized by $v\left(a, i_{t}\right)=1 / 2$ and equals

$$
\frac{1}{2}\left[\max _{x} f\left(y_{t}^{\alpha}(x)\right)+\max _{x} f\left(y_{t}^{\beta}(x)\right)\right]+h\left(\frac{1}{2}\right) .
$$

Therefore, if no activity dominates the other one in terms of income irrespective of the state of the world, the optimal socialization strategy could be to allocate an equal amount of symbolic value to each activity. This diversification strategy could never be optimal in the deterministic model. Diversification actually is optimal if the child's expected utility is larger in that case rather than in the specialization case, i.e., if

$$
\frac{1}{2}\left[\max _{x} f\left(y_{t}^{\alpha}(x)\right)+\max _{x} f\left(y_{t}^{\beta}(x)\right)-\max _{x}\left\{f\left(y_{t}^{\alpha}(x)\right)+f\left(y_{t}^{\beta}(x)\right)\right\}\right]>h(1)-h\left(\frac{1}{2}\right) .
$$

Turning to the general case, where parameter $\eta \in(0,1]$, we establish

Proposition 4 Assume $\sigma>1$. If $\chi$ is large enough, then the the economy behaves has a value-less one and the value systems of all families converge to $\{1 / 2,1 / 2\}$.

Proof: At any $t$, individual $i_{t}$ surely chooses the occupation with the largest income, irrespective of his value system, if

$$
\min _{\omega}\left|f\left(y_{t}^{\omega}(a)\right)-f\left(y_{t}^{\omega}(b)\right)\right|>\left|h\left(v\left(a, i_{t}\right)\right)-h\left(1-v\left(a, i_{t}\right)\right)\right| .
$$

If $\chi \rightarrow 1$, then $y_{t}^{\beta}(a) \rightarrow 0$ and $y_{t}^{\alpha}(b) \rightarrow 0$. Then, because $\sigma>1$, one has $f\left(y_{t}^{\beta}(a)\right) \rightarrow$ $-\infty$ and $f\left(y_{t}^{\alpha}(b)\right) \rightarrow-\infty$. It follows that

$$
\lim _{\chi \rightarrow 1} \min _{\omega}\left|f\left(y_{t}^{\omega}(a)\right)-f\left(y_{t}^{\omega}(b)\right)\right|=+\infty,
$$


so that if $\chi$ is large enough, individual $i_{t}$ chooses the occupation with the largest income independently of his values. By setting $\chi$ arbitrarily close to 1 , condition (15) is met for all families and all periods. Then, in equilibrium the arbitrage condition $y_{t}(a)=y_{t}(b)$ applies for all $t$, implying that the economy behaves exactly as in the absence of values.

If $\chi$ is so large that values do no affect occupational choice, individual $i_{t-1}$ chooses $v\left(a, i_{t}\right)$ in the interval $\left[\underline{v}\left(a, i_{t}\right), \bar{v}\left(a, i_{t}\right)\right]$ so as to maximize the child's expected utility from self-esteem, which is given by

$$
\frac{1}{2}\left[h\left(v\left(a, i_{t}\right)\right)+h\left(1-v\left(a, i_{t}\right)\right)\right] .
$$

The solution is to set $v\left(a, i_{t}\right)$ as close as possible to $1 / 2$ subject to the constraint that it must be in the interval $\left[\underline{v}\left(a, i_{t}\right), \bar{v}\left(a, i_{t}\right)\right]$, that is $v\left(a, i_{t}\right)=1 / 2$ if $1 / 2 \in\left[\underline{v}\left(a, i_{t}\right), \bar{v}\left(a, i_{t}\right)\right]$ and $v\left(a, i_{t}\right)=\underline{v}\left(a, i_{t}\right)$ if $\underline{v}\left(a, i_{t}\right)>1 / 2, v\left(a, i_{t}\right)=\bar{v}\left(a, i_{t}\right)$ if $\bar{v}\left(a, i_{t}\right)<1 / 2$. It follows that $\left|v\left(a, i_{t}\right)-1 / 2\right| \leq\left|v\left(a, i_{t-1}\right)-1 / 2\right|$ and that the value system of every family converges to $\{1 / 2,1 / 2\}$. QED

Hence, provided that the predictability of the future incomes from the two occupations is low, the equilibrium can be consistent with the materialistic view even if the intertemporal elasticity of substitution of consumption is smaller than one. The intuition is straightforward. If the amount of uncertainty is large, the income differential between the two occupations is also large and if this differential increases, at some point an individual will choose the occupation with the higher income, irrespective of his values. ${ }^{12}$ Anticipating this, the individual's parent will diversify the individual's values, thereby contributing to insure the individual's esteem. Hence, it is risk aversion with respect to esteem, or concavity of $h$, that can prompt parents to select a diversifed value system for their children.

If $\chi>0$ but not so large that material rewards are overwhelming, values can still matter for long-run growth if the intertemporal elasticity of substitution of consumption is smaller than one. However, differently from the deterministic case, in the stochastic case families do not necessarily specialize themselves in the long run. Specifically, we establish the following fact:

Proposition 5 Assume $f(y)=\log (y)$. Let the population at time 0 consist of three groups: $n_{a}$ individuals who put all the value in occupation $a, n_{b}$ individuals who put all the value in occupation $b$, and $n_{a b}$ individuals who attach the same value $1 / 2$ to both occupations. Then, there can exist intervals for $n_{a}, n_{b}$ and $n_{a b}$ for which this distribution

\footnotetext{
${ }^{12}$ This also holds for $f(\cdot)$ logarithmic, which means that Proposition 4 carries over to $\sigma=1$.
} 
of values persists over time. The growth rate is stochastic and its average level increases with $n_{a}$.

Proof: Fix strictly positive numbers $n_{a}, n_{b}$ and $n_{a b}$ that satisfy

$$
\begin{aligned}
& (1-\chi) Y_{a}\left(n_{a}\right)<(1+\chi) Y_{b}\left(n_{b}+n_{a b}\right) \\
& (1-\chi) Y_{b}\left(n_{b}\right)<(1+\chi) Y_{a}\left(n_{a}+n_{a b}\right)
\end{aligned}
$$

and $n_{a}+n_{b}+n_{a b}=1$. The families that put all symbolic value in occupation $a$ and always practice that occupation never have an incentive to deviate if

$$
\log \left[\frac{(1+\chi) Y_{b}\left(n_{b}+n_{a b}\right)}{(1-\chi) Y_{a}\left(n_{a}\right)}\right] \leq h(1)-h(\eta) .
$$

A symmetric condition ensures that all the families that put all symbolic value in occupation $b$ and always practice that occupation never have an incentive to deviate:

$$
\log \left[\frac{(1+\chi) Y_{a}\left(n_{a}+n_{a b}\right)}{(1-\chi) Y_{b}\left(n_{b}\right)}\right] \leq h(1)-h(\eta) .
$$

The remaining families never have an incentive to deviate if $\operatorname{Inf}\left\{\log \left[\frac{(1+\chi) Y_{b}\left(n_{b}+n_{a b}\right)}{(1-\chi) Y_{a}\left(n_{a}\right)}\right], \log \left[\frac{(1+\chi) Y_{a}\left(n_{a}+n_{a b}\right)}{(1-\chi) Y_{b}\left(n_{b}\right)}\right]\right\} \geq h\left(\frac{1}{2}+\eta\right)-h\left(\frac{1}{2}-\eta\right)$.

To sum up, all strictly positive numbers $n_{a}, n_{b}$ and $n_{a b}$ that sum to 1 and satisfy (16) and (17) describe an equilibrium configuration if they also satisfy (18) and

$$
h(1)-h(\eta) \geq \operatorname{Sup}\left\{\log \left[\frac{(1+\chi) Y_{b}\left(n_{b}+n_{a b}\right)}{(1-\chi) Y_{a}\left(n_{a}\right)}\right], \log \left[\frac{(1+\chi) Y_{a}\left(n_{a}+n_{a b}\right)}{(1-\chi) Y_{b}\left(n_{b}\right)}\right]\right\} .
$$

It is easy to verify that there exist parameter constellations such that for each constellation there exists a set of triples $\left(n_{a}, n_{b}, n_{a b}\right)$ that fulfill all conditions.

The equilibrium growth rate is then stochastic and its expected value is given by

$$
\frac{1}{2}\left[g\left(1-n_{b}\right)+g\left(n_{a}\right)\right]
$$

Increasing $n_{a}$ necessarily increases the expected growth rate. QED

Uncertainty about income opportunities can make families diversify their values. Increasing uncertainty beyond a certain point generates cultural convergence, i.e. all families 
eventually share the same value system and the esteem differential between the occupations vanishes. This finding suggests an interpretation for the diminished role of professional pride in modern as compared to some traditional societies. The pronounced craft honor in medieval towns, e.g. goldsmiths, barbers and merchants in Italian free city states, was likely to be supported by strong confidence in professional continuity along familiy lines, which gave parents an incentive to invest in the symbolic value of their own occupation. Conversely, the industrial revolution and the liberalization of markets for professions implied a large degree of occupational mobility and a lower degree of predictability of future economic activities. This may have dissuaded parents to invest symbolic value unilaterally in their own occupation, since this would have created an impediment for their children to fully take advantage of new economic opportunities.

\section{Social esteem}

Concerns for social esteem are likely to influence occupational choice and in this way to affect the growth prospects of an economy. The concept of symbolic value introduced above allows one to think about the social esteem that individuals enjoy because they practice a given occupation. The social esteem in which an individual is held may be defined as the average of the esteem granted to his activity over the whole society:

$$
\operatorname{socv}\left(x\left(i_{t}\right)\right)=\int_{0}^{1} v\left(x\left(i_{t}\right), j\right) d j .
$$

We may then write individual utility as

$$
U\left(i_{t}\right)=f\left(y_{t}\left(x\left(i_{t}\right)\right)\right)+h\left(v\left(x\left(i_{t}\right), i_{t}\right)+\gamma z\left(\operatorname{socv}\left(x\left(i_{t}\right)\right)\right),\right.
$$

where $z^{\prime}>0$ and $\gamma \geq 0$ captures the strength of the concern for social esteem. This parameter may be thought of as reflecting both psychological predispositions and the intensity of social interactions. If individuals enter frequent interactions with a relatively small number of people over most of their lifetime, like in a traditional village, then one might assume that $\gamma$ is larger than in the case of a loose network of anonymous, short-lived, contacts, like in a modern city.

The presence of social esteem generates an externality in the choice of values: while parents choose values so as to maximize their children's expected utility, they directly affect the esteem that other children will receive. This externality entails a distinctive mechanism by which value systems and economic development interact, one that was absent in the model where the only symbolic reward of occupations comes in form of self-esteem. 
To illustrate the distinctive implications of a concern for social esteem, we embed (19) and (20) in the deterministic model of Sections 4-6. It is easy to verify that Propositions 2 and 3, derived under the assumption that $\eta$ is small, carry over to the model with social esteem. That is, values matter for long-run growth if and only if the intertemporal elasticity of substitution of consumption is smaller than one. However, if individuals care about social esteem, a long-run effect of values can also exist if $\eta=1$, i.e., if parents are not constrained in the choice of their children's values.

To establish that result, consider the short-run equilibrium of the model with social esteem and $\eta=1$. In such an equilibrium, (5) and (6) apply with $\bar{v}\left(a, i_{t+1}\right)=1$ and $\underline{v}\left(a, i_{t+1}\right)=0$. Then, the self-esteem associated with occupations $a$ and $b$ are respectively given by 1 and 1 and the corresponding social esteems are $n_{t+1}$ and $1-n_{t+1}$. It follows that the net benefit of occupation $a$ relative to occupation $b$ is

$$
B_{t+1}\left(n_{t+1}\right)=\left[f\left(A_{t+1} Y_{a}\left(n_{t+1}\right)\right)-f\left(A_{t+1} Y_{b}\left(n_{t+1}\right)\right)\right]+\gamma\left[z\left(n_{t+1}\right)-z\left(1-n_{t+1}\right)\right] .
$$

The first term in square brackets on the right-hand side of this equation is decreasing with $n_{t+1}$ because the difference between the income of type $a$ individuals and type $b$ individuals decreases with the relative number of type $a$ individuals. The second term shows that the relative social esteem granted to occupation $a$ is increasing with the number of individuals who value this occupation, $n_{t+1}$. If $\gamma$ is large enough, the second term dominates the first one, implying that there are two stable short-run equilibria, one in which all individuals practice $a$ and one in which they all practice $b$.

Thus, a concern for social esteem can lead to conformism. By choosing to invest symbolic value in the future occupation of his offspring, an individual reduces the social esteem for the other occupation and thus induces other individuals to imitate him. This may generate a bandwagon effect in the formation of value systems and the choice of occupations. $^{13}$

Proposition 6 Assume $\eta=1$. Let the entire population at time 0 put all the value in the same occupation and practise that occupation. There is an equilibrium in which this state of affairs persists for ever if and only if $\sigma>1$.

\footnotetext{
${ }^{13}$ This phenomen was apparently observed by Blaise Pascal in the middle of the 17th century, when he noted: "La chose la plus importante à toute la vie, est le choix du métier: le hasard en dispose. La coutume fait les macons, soldats, couvreurs. "C'est un excellent couvreur", dit-on; et, en parlant des soldats: "Ils sont bien fous", dit-on; et les autres au contraire: "Il n'y a rien de grand que la guerre; le reste des hommes sont des coquins". A force d'ouir louer en l'enfance ces métiers, et mépriser tous les autres, on choisit; ... car des pays sont tous de macons, d'autres tous de soldats, etc. Sans doute que la nature n'est pas si uniforme. C'est la coutume qui fait donc cela..." (Pensées et Opuscules, Larousse, Paris, 39th ed., 1934, p. 28-29).
} 
Proof: Suppose without loss of generality that the population is specialized in occupation $b$. Then,

$$
f\left(A_{0} Y_{b}(1)\right)+\gamma z(1) \geq f\left(A_{0} Y_{a}(0)\right)+\gamma z(0)
$$

must hold in the initial short-run equilibrium. This state of affairs can persist if

$$
\gamma[z(1)-z(0)] \geq A_{t}^{1-\sigma}\left[Y_{a}(0)-Y_{b}(1)\right]
$$

for all $t>0$. Since $n^{*} \in(0,1)$, it must be the case that $Y_{a}(0)>Y_{b}(1)$. Then, condition (21) is always met as $A_{t}$ increases to $+\infty$ if and only if $\sigma>1$. QED

Multiple value-led equilibria can persist indefinitely if the intertemporal elasticity of substitution of consumption is smaller than one. ${ }^{14}$ If $\gamma$ is large enough, there exists both an equilibrium with a long-run growth rate equal to $g(0)$ and one with with a long-run growth rate equal to $g(1)$. Of course, $g(1)>g\left(n^{*}\right)>g(0)$, so that values matter in an extreme form for long-term growth in this case.

Notice, however, that the interpretation of the current result is different from the one in the model with no social esteem $(\gamma=0)$ and $\eta$ small. In the current framework, values do not matter in the sense that their initial constellation shapes the long-run behavior of the economy. They matter because the expectation of a certain constellation of values in the future and the desire to avoid their offspring incurring ostracism lead parents of all generations to inculcate values that validate that expectation.

Proposition 6 suggests that circumstances can exist under which values are responsible for big differences in development between economies with small differences in technology. Consider the case of two identical countries in a long-run equilibrium where everybody performs activity $b$, the traditional activity, and $g(0) \simeq 0$. Now conceive a development opportunity in the following sense: at some point in time, a technology shock raises the income level that can be obtained from activity $a$, the modern activity, i.e., function $Y_{a}(\cdot)$ shifts upwards in both countries. If the improvement of opportunities differs in the two countries, condition (21) may be satisfied after the shock in only one country. While culture and income will not change in this country, the other one will experience a cultural revolution and an economic take-off.

\section{Conclusion}

The endogenous growth model developed in this paper offers a simple theoretical framework for addressing the old question of the interaction between economic development

\footnotetext{
${ }^{14}$ As it is easily checked, they can also persist in the logarithmic case.
} 
and value systems. People's economic activity typically results from the deliberate choice to practice a distinctive occupation, often for the entire duration of one's economically active life. For most people, work is one defining element of the self, not simply because a large fraction of one's lifetime is absorbed by work but also because it is mainly through work that the person consciously shapes her environment, i.e., expresses her individuality. Therefore, economic activity is a central category for defining one's identity and a natural object of value judgements.

Our model explores the economic implications of the idea that people can invest in the value of occupations, i.e., they can influence their children's evaluations of occupations. Specifically, we have derived the evolution of value systems that arises when parents select them so as to maximize their children's expected utility. In our framework, economic variables and value systems mutually affect each other. On the one hand, the path of income opportunities associated with the various occupations affects the values transmitted by parents to children. On the other hand, both one's acquired values and others' evaluations of occupations affect one's choice of economic activity; in this way, value systems affect the growth prospects of the entire economy.

Symbolic values can have a long-term effect on economic growth in our model. The culturalist view of development is more likely to be correct if the intertemporal elasticity of substitution of consumption is low, predictability of the economic environment is high, and concern for social ostracism is strong. In those cases, culturally determined poverty traps can exist. Under different circumstances, the materialistic view may be the correct one: technological change can be disruptive of value systems that existed for a long time and entail a process of cultural convergence.

The model in this paper may be extended in several directions. Given its stress on endogenous value formation, a further exploration in this area would be warranted. Values are not only transmitted from one generation to the next, but also within generations. This horizontal socialization occurs in society at large via imitation and learning from peers and role models; "oblique transmission" occurs when values are acquired from nonparental adults. ${ }^{15}$

Horizontal socialization could be introduced in our model alongside vertical socialization. Define for each young individual a probability distribution over value systems: the individual's actual value system may be assumed to be randomly selected according to that probability distribution. The probabilities associated with the various value systems

\footnotetext{
${ }^{15}$ Models of horizontal socialization were pioneered by evolutionary anthropologists Cavalli-Sforza and Feldman (1981) and Boyd and Richerson (1985), who exploited analogies between cultural transmission and epidemics. The purposive aspect of cultural transmission was introduced later by Bisin and Verdier (2000, 2001).
} 
may be assumed to respond to both the values taught by parents and the values endorsed by society at large. More realistically, reference groups could be defined from which an individual is relatively likely to acquire values.

Following Bisin and Verdier's approach, one may further assume that socialization by parents is costly, and that parents can increase the probability of determining their children's values by investing more resources in socializing them. This ingredient may produce further insights into the value system of a society. To the extent that vertical socialization requires parents to spend time with their children, a substitution effect might dominate by which more productive parents spend less time with their children and the social esteem of highly productive occupations is relatively low. If vertical socialization can be bought - e.g. services of private teachers and clubs are used to influence the children's values - an income effect may dominate so that wealthier parents are more likely to shape values. Then, the values of the affluent would tend to be overrepresented in society. 


\section{APPENDIX}

\section{A microfoundation of Equations (3) and (4).}

There is a traditional and a modern sector and the two activities produce two different goods, denoted by $a$ and $b$. Utility of consumption is Cobb-Douglas, i.e. it increases with the aggregate consumption index

$$
c=\left(\frac{c_{a}}{\alpha}\right)^{\alpha}\left(\frac{c_{b}}{1-\alpha}\right)^{1-\alpha} .
$$

An income $y$ allows an individual to buy a quantity $y / p$ of composite good, with the consumption price index given by

$$
p=p_{a}^{\alpha} p_{b}^{1-\alpha},
$$

where $p_{a}$ and $p_{b}$ are the prices of goods $a$ and $b$ respectively.

An individual produces a quantity $\widetilde{A}_{t} q_{a}$ at time $t$ if he is active in sector $a$. The productivity level in sector $b$ is constant over time and given by $q_{b}$ units of good.

Market prices are competitively determined. The Cobb-Douglas assumption implies,

$$
\frac{n p_{a} \widetilde{A} q_{a}}{(1-n) p_{b} q_{b}}=\frac{\alpha}{1-\alpha} .
$$

Using (22) and the expression above to substitute out the relative price $p_{a} / p_{b}$ implies that the real incomes in the two sectors are given by

$$
y_{a}=p_{a} \widetilde{A} q_{a} / p=\alpha q \widetilde{A}^{\alpha}\left(\frac{1-n}{n}\right)^{1-\alpha}
$$

and

$$
y_{b}=(1-\alpha) q \widetilde{A}^{\alpha}\left(\frac{n}{1-n}\right)^{\alpha},
$$

where $q$ is a shorthand for $\left(q_{a} / \alpha\right)^{\alpha}\left(q_{b} /(1-\alpha)\right)^{1-\alpha}$. Then, the incomes to activities $a$ and $b$ can be written as

$$
\begin{gathered}
Y_{a}(n)=\alpha q\left(\frac{1-n}{n}\right)^{1-\alpha}, \\
Y_{b}(n)=(1-\alpha) q\left(\frac{n}{1-n}\right)^{\alpha},
\end{gathered}
$$

and the productivity parameter is given by

$$
A_{t}=\widetilde{A}_{t}^{\alpha} .
$$

Notice that even though productivity grows in the modern sector only, income grows at the same rate in the two sectors because of the increase in the relative price of the traditional good. 


\section{References}

Aghion, P. and P. Howitt, 1992, A Model of Growth through Creative Destruction, Econometrica 60, 323-351.

Akerlof, G. and R. Kranton, 2000, Economics and Identity, Quarterly Journal of Economics 115, 715-753.

Akerlof, G. and R. Kranton, 2005, Identity and the Economics of Organizations, Journal of Economic Perspectives 19, 9-32.

Baumol, W., 1990, Entrepreneurship: Productive, Unproductive, and Destructive, Journal of Political Economy 98, 893-921.

Bénabou R. and J. Tirole, 2006, Identity, Dignity and Taboos: Beliefs as Assets, mimeo Princeton University and University of Toulouse.

Bisin, A. and T. Verdier, 2000, Beyond the Melting Pot: Cultural Transmission, Marriage, and the Evolution of Ethnic and Religious Traits, Quarterly Journal of Economics, 955-988.

Bisin, A. and T. Verdier, 2001, The Economics of Cultural Transmission and the Dynamics of Preferences, Journal of Economic Theory, 97, 298-319.

Boyd, R., and P. Richerson, 1985, Culture and the Evolutionary Process, Chicago: University of Chicago Press.

Cavalli-Sforza, L. and M. Feldman, 1981, Cultural Transmission and Evolution: A Quantative Approach, Princeton: Princeton University Press.

Cole, H., Mailath, G., and A. Postlewaite, 1992, Social Norms, Savings Behavior, and Growth, Journal of Political Economy 100, 1092-1125.

Corneo G. and O. Jeanne, 2006, A Theory of Tolerance, CEPR Discussion Paper No. 5908.

Fernandez, R., 2007, Women, Work, and Culture, NBER Working Paper No. 12888.

Fershtman, C., Murphy, K. and Y. Weiss, 1996, Social Status, Education, and Growth, Journal of Political Economy 104, 108-132.

Fershtman, C. and Y. Weiss, 1998a, Social Status and Economic Performance: A Survey, European Economic Review 42, 801-820. 
Fershtman, C. and Y. Weiss, 1998b, Why Do We Care what Others Think about Us?, in Ben-Ner, A. and L. Putterman (eds.), Economics, Values, and Organizations, Cambridge University Press.

Francois, Patrick and Jan Zabojnik, 2005, Trust, Social Capital and Economic Development, Journal of the European Economic Association 3, 51-94.

Guiso, L., Sapienza, P. and L. Zingales, 2006, Does Culture Affect Economic Outcomes?, NBER Working Paper No. 11999.

Inglehart, R. and W. Baker, 2000, Modernization, Cultural Change, and the Persistence of Traditional Values, American Sociological Review 65, 19-51.

Keynes, J. M., 1972, Economic Possibilities for Our Grandchildren, in Essays in Persuasion (first edition: 1931), Macmillan.

Murphy, K., Shleifer, A. and R. Vishny, 1991, The Allocation of Talent: Implications for Growth, Quarterly Journal of Economics 106, 503-530.

Romer, P. M., 1990, Endogenous Technological Change, Journal of Political Economy 98, 71-102.

Tabellini, G., 2006, Culture and Institutions: Economic Development in the Regions of Europe, mimeo, IGIER. 